\title{
Divergencias en la percepción de prácticas parentales, comportamiento positivo y problemáticas entre padres e hijos
}

\author{
María del Pilar Méndez Sánchez; Rafael Peñaloza Gómez; Mirna García Méndez; \\ José Carlos Jaenes Sánchez; Héctor Rafael Velázquez Jurado
}

How to cite this article:

Méndez, M.P., Peñaloza, R., García, M., Jaenes, J.C., \& Velázquez, H.R. (2019). Divergences in the perception of parental practices, positive behavior and problems between parents and children. Acta Colombiana de Psicología, 22(2), $194-205$. doi: http://www.doi.org/10.14718/ACP.2019.22.2.10

Recibido, abril 20/2018; Concepto de evaluación, septiembre 17/2018; Aceptado, enero 16/2019

\author{
María del Pilar Méndez Sánchez* \\ Universidad Nacional Autónoma de México, México \\ ORCID: https://orcid.org/0000-0003-0066-5582 \\ Rafael Peñaloza Gómez \\ Universidad Nacional Autónoma de México, México \\ ORCID: https://orcid.org/0000-0003-3585-7701 \\ Mirna García Méndez \\ Universidad Nacional Autónoma de México, México \\ ORCID: https://orcid.org/0000-0002-2334-0740 \\ José Carlos Jaenes Sánchez \\ Universidad Pablo de Olavide, Sevilla, España \\ ORCID: https://orcid.org/0000-0002-8700-130X \\ Héctor Rafael Velázquez Jurado \\ Instituto Nacional de Ciencias Médicas y Nutrición, Salvador Zubirán, México \\ ORCID: https://orcid.org/0000-0002-7660-9336
}

Resumen

\begin{abstract}
El presente estudio tuvo como finalidad analizar las divergencias en la percepción de las prácticas parentales, el comportamiento positivo y las problemáticas entre padres e hijos. Los participantes, padres y adolescentes, colaboraron de manera voluntaria y se evaluaron en las siguientes díadas: 248 padres-hijos, 241 padres-hijas, 237 madres-hijos, y 241 madres-hijas. Los instrumentos utilizados fueron la Escala de Capacidades y Dificultades y la Escala de Prácticas Parentales para madres y padres. Para lograr el objetivo principal se realizaron análisis de correlación, análisis de senderos y diferencias de medias con muestras relacionadas. Los resultados mostraron correlaciones - de moderadas a bajas- entre las prácticas parentales, los problemas y la conducta prosocial, además de diferentes factores predictores de las conductas de los hijos a partir del sexo de los padres y las prácticas parentales ejercidas. Se presentaron divergencias entre los reportes de prácticas parentales, conducta prosocial y problemas entre los padres y sus hijos. Se concluye que la conducta parental se orienta según el sexo de los hijos, lo cual implica cierta deseabilidad social relacionada con su rol como hombres o como mujeres.

Palabras clave: prácticas parentales, problemas de conducta, conducta prosocial, familia, comunicación.
\end{abstract}

\section{Divergences in the perception of parental practices, positive behavior and problems between parents and children}

\begin{abstract}
The purpose of this study was to analyze the divergences in perception of parental practices, positive behavior and problems between parents and children. The participants, parents and adolescents, collaborated voluntarily and were examined in the following dyads: 248 fathers-sons, 241 fathers-daughters, 237 mothers-sons, and 241 mothers-daughters. The psychometric
\end{abstract}

\footnotetext{
Facultad de Estudios Superiores Zaragoza, Universidad Nacional Autónoma de México, Campus I, Av. Guelatao No. 66 Col. Ejército de Oriente, Iztapalapa C.P. 09230, Ciudad de México. Carrera de Psicología. Número Telefónico+5559158182.pilar_mendez@comunidad. unam.mx
} 
instruments used were the Strengths and Difficulties Scale and the Parental Practices Scale for mothers and fathers. To achieve the main objective, correlation analysis, path analysis and differences of means with related samples were performed. The results showed correlations, from moderate to low, between parental practices, problems and prosocial behavior. In addition, different predictive factors of the children's behaviors were obtained, according to the sex of the parents and the parental practices they implemented. Finally, there were divergences between reports of parental practices, prosocial behavior and problems between parents and their children. Therefore, it is concluded that parental behavior is oriented according to the sexual role of their children, which involves social desirability.

Key words: parental practices, behavior problems, prosocial behavior, family, communication.

\title{
Divergências na percepçáo de práticas parentais, comportamento positivo e problemáticas entre pais e filhos
}

\begin{abstract}
Resumo
O presente estudo teve como finalidade analisar as divergências na percepção das práticas parentais, no comportamento positivo e nas problemáticas entre pais e filhos. Os participantes, pais e adolescentes, colaboraram de maneira voluntária e foram avaliados nas seguintes díades: 248 pais-filhos, 241 pais-filhas, 237 mães-filhos e 241 mães-filhas. Os instrumentos utilizados foram a Escala de Capacidades e Dificuldades e a Escala de Práticas Parentais para mães e pais. Para alcançar o objetivo principal, realizaram-se análises de correlação, análises de caminhos e diferenças de médias com amostras relacionadas. Os resultados mostraram correlações — de moderadas a baixas — entre as práticas parentais, os problemas e a conduta pró-social, além de diferentes fatores preditivos das condutas dos filhos a partir do gênero dos pais e das práticas parentais exercidas. Houve divergências entre os relatórios de práticas parentais, conduta pró-social e problemas entre os pais e seus filhos. Conclui-se que a conduta parental é orientada de acordo com o gênero dos filhos, o que implica certa desejabilidade social relacionada com seu papel como homens ou como mulheres.

Palavras-chave: práticas parentais, problemas de conduta, conduta pró-social, família, comunicação.
\end{abstract}

\section{Introducción}

La Organización Mundial de Salud (OMS; 2018) define la adolescencia como el periodo de crecimiento y desarrollo humano que se produce entre los $10 \mathrm{y}$ los 19 años de edad. En México, el 9.3 \% de la población en el 2015 se encontraba entre dichas edades, es decir, se calculaban aproximadamente 11154278 de adolescentes en el país (INEGI, 2015).

Como informa la OMS (2018), al final de la infancia o comienzo de la adolescencia suelen aparecer numerosos problemas de salud mental que pueden tener efectos importantes en la salud en general y en el desarrollo del adolescente. Por ejemplo, estudios como el de Díaz y González (2014), a partir de reportes de adolescentes de entre 14 y 19 años de la ciudad de Monterrey (México), muestran la presencia de consumo de cigarros, alcohol y mariguana, (Díaz \& González, 2014); al igual que el estudio de Hernández, Guadarrama, Veytia y Márquez (2017), en donde se encuentran puntajes significativos de depresión, problemas somáticos, problemas de pensamiento, rompimiento de reglas, lesiones autoinfligidas y conducta agresiva en adolescentes mexicanos de edades entre los 15 y los 18 años.

Teniendo en cuenta las diferencias entre hombres y mujeres, se ha encontrado evidencia de que los hombres presentan más problemas externalizados y de conducta -como agresividad, inquietud, desobediencia y burlas-que las mujeres, quienes reportan más problemas internalizados y emocionales -como sentimientos de inferioridad, soledad, tristeza, ansiedad (Bailey, Zauszniewski, Heinzer \& Hemstrom-Krainess, 2007; Méndez, Andrade \& Peñaloza, 2013), síntomas depresivos, problemas somáticos y lesiones autoinfligidas (Betancourt \& Andrade, 2011)-; además de que los problemas ansiosos son más frecuentes en las mujeres, mientras que el trastorno por déficit de atención con hiperactividad se presenta más en los hombres (De la Peña-Olvera, Gómez, Heinze \& Palacios-Cruz, 2014). Estos resultados confirman lo que se entiende como deseabilidad social, es decir, que en las mujeres se espera un comportamiento más pasivo, discreto, apacible y amable, mientras que en el hombre se espera más actividad o agresividad y que sea dominante, ambicioso, abierto e independiente.

Hay que destacar que en los estudios que utilizaron el instrumento de evaluación SDQ (Strengths \& Difficulties Questionnaire) -cuestionario retomado para la presente investigación- reportaron que las mujeres presentan más problemas emocionales, mientras que los hombres manifiestan más problemas de conducta, hiperactividad, falta de atención y problemas con sus compañeros (Giannakopoulos et al., 2009; Schojaei, Wazana, Pitrou \& Kovess, 2009); sin embargo, un estudio en Pakistán (Syed, Hussein \& Mahmud, 2007) no 
196

señaló diferencias entre hombres y mujeres en la dimensión de problemas emocionales y problemas con compañeros, pero sí en problemas de conducta e hiperactividad y falta de atención, al igual que los otros estudios en los que se encontró que los hombres presentan más este tipo de conducta.

Por lo que refiere a los padres, se ha encontrado que por medio de su educación inculcan en sus hijos habilidades para su inserción posterior al contexto social mediante prácticas parentales (Campos, Eceiza \& Paéz, 2004), definidas como dominios específicos de conductas relacionadas con el control psicológico, el control conductual, el monitoreo y la comunicación, que funcionan como mecanismos de relación entre padres e hijos para que el niño o adolescente alcance ciertas metas de socialización (Darling \& Steinberg, 1993; Smetana, 2017).

En este sentido, se puede afirmar que las conductas parentales influyen directamente en el desarrollo de los hijos, y es por esto que las condiciones de adversidad familiar se asocian con el desarrollo de conductas desadaptativas internalizadas y externalizadas en niños y adolescentes (Coln, Jordan \& Mercer, 2013). Así, por ejemplo, si los padres son controladores, exigentes y hostiles en la educación, sus hijos manifiestan una baja autoestima, más agresividad, hostilidad e impaciencia (Castro, 2005).

En particular, en México se reportó que un alto control psicológico de ambos padres y una baja supervisión paterna influyeron en problemas depresivos, problemas de pensamiento, lesiones autoinfligidas y problemas externalizados de adolescentes hombres, mientras que en las mujeres se encontraron problemas depresivos y somáticos relacionados con un alto control psicológico de ambos padres y poca supervisión del padre, así como problemas de pensamiento relacionados con una menor comunicación con la madre y un alto control psicológico del padre (Betancourt \& Andrade, 2011). Además de esto, la conducta de los padres también influye en la conducta prosocial que desarrollan sus hijos, ya que, ante el desinterés parental, negligencia o control patológico, se presenta menor conducta prosocial (Richaud de Minzi, Lemos \& Mesurado, 2011). En consecuencia, cuando los niños crecen en ambientes en los cuales son frecuentemente maltratados, existe un mayor riesgo para desarrollar modelos mentales de las relaciones sociales que incorporen ese maltrato (Álvarez, 2010).

Por el contrario, se ha encontrado que el afecto-comunicación, la promoción de la autonomía y el humor se relacionan con un mejor ajuste interno y externo del adolescente (Oliva, Parra, Sánchez-Queija \& López, 2007); que la percepción de una elevada aceptación paterna junto con un bajo control patológico y una baja autonomía extrema propician la tranquilidad (Meier \& Oros, 2012); y que el reporte de las prácticas de crianza basadas en el reforzamiento positivo, el control de estímulos, la corrección simple del comportamiento y la técnica de ignorar la conducta no deseada se relacionan con un menor porcentaje de reportes de conducta agresiva -negativista o desafiante-(Morales, Félix, Rosas, López \& Nieto, 2015). Por lo tanto, se demuestra que es más probable encontrar un mejor ajuste en los adolescentes cuando los padres supervisan las actividades de sus hijos y cuentan con un estilo de educación democrático (GómezOrtiz, Del Rey, Romera \& Ortega-Ruiz, 2015).

Además de esto, se ha encontrado que los problemas propios de los padres se vinculan con la conducta parental y con el trato que se tiene ante los hijos, es decir, que los síntomas depresivos de los padres -por ejemplo-influyen en los síntomas internalizados y externalizados de sus hijos (Reising et. al., 2013). En este sentido, algunos estudios han señalado que los síntomas depresivos se relacionan con un bajo cuidado, rechazo y pobre monitoreo, lo cual impacta en la presencia de problemas en los niños (Elgar, Mills, McGrath, Waschbusch \& Brownridge, 2007); y que estos síntomas, junto con los pensamientos negativos y el estrés crónico de la madre, influyen en la presencia de distintos problemas de carácter psicológico en sus hijos (Davé, Sherr, Senior \& Nazareth, 2008; Hall, Rayens \& Peden, 2008).

Por otra parte, en lo que se refiere a las expectativas de los padres, se han identificado comportamientos relacionados con la deseabilidad social, en donde los padres esperan que sus hijos e hijas se comporten de manera determinada a partir de una serie de perspectivas y atribuciones sociales, por lo que madres y padres tienen comportamientos diferentes con sus hijos en función del sexo. Así, las madres se acercan más a sus hijos e hijas cuando hay una situación femenina que ante una situación masculina, y mantienen mayor número de interacciones -aunque mayor con las hijas que con los hijos-, mientras que los padres se muestran más distanciados de sus hijos e hijas (Rocha, 2004).

Ahora bien, con respecto a las discrepancias en la percepción de las prácticas de crianza y los conflictos familiares entre padres e hijos es importante destacar: (a) que durante la adolescencia se presentan diversas crisis del desarrollo que hacen que el individuo enfrente conflictos ocasionados por cambios físicos, emocionales y cognitivos -que pueden ser producidos por el sujeto, el contexto o la relación entre ambos- (Papalia \& Martorell, 2017); y (b) que cuando el adolescente se encuentra en confusión y conflicto se despierta cierto rechazo hacia sus padres, manifestado en cambios en la comunicación y desobediencia, lo cual puede conllevar a castigos por parte de los padres o bien a conductas agresivas donde se ve afectada la unión intrafamiliar (Urbano \& Yuni, 2016).

Al respecto, se pueden identificar diversas manifestaciones de las discrepancias entre la percepción de las 
prácticas parentales y las conductas entre el adolescente y sus padres. Por ejemplo, Aguilar-Morales, Aguilar-Morales y Vargas-Mendoza (2006) mencionan que algunos padres reportan conductas consideradas como problemáticas, aunque estas no impliquen la presencia de trastornos de comportamiento, por lo que indican que es un error común tanto patologizar aquellas conductas que resultan inconvenientes para los padres como no considerar los reportes de los niños. Al no tener en cuenta estos errores, gran parte de los diagnósticos se establecen por las complicaciones que genera la conducta de los niños en los padres y no por el sufrimiento reportado por los hijos.

Adicionalmente, a partir las diferencias según el sexo de los padres, en algunos estudios se reportó que los adolescentes tienen más conflictos con las madres que con los padres, posiblemente porque pasan mayor tiempo con ella, porque muchos de los conflictos tienen que ver con la vida cotidiana (Parra \& Oliva, 2002), o porque ellas son más conscientes que el padre de la frecuencia e intensidad emocional de algunos conflictos en las relaciones con los hijos e hijas (Rodrigo, García, Máiquez \& Triana, 2005). También, aunque se reportó que las madres tienen más comunicación con sus hijos, que muestran más interés por las sus experiencias y que están más pendientes de las cosas que hacen (Oudhof, Rodríguez \& Robles, 2012), también se encontró que la comunicación es más fluida cuando se presentan las díadas padre-hijo y madre-hija (Rodríguez, Del Barrio \& Carrasco, 2009; Sevilla, Sanabria, Orcasita \& Palma, 2016). En una investigación se encontró que en las familias en las que la madre es más conocedora de las actividades de sus hijos, y el padre es poco conocedor, los adolescentes muestran conductas de riesgo como ingesta de alcohol y cannabis (Jiménez-Iglesias \& Moreno, 2015).

Teniendo lo anterior en cuenta, la presente investigación tuvo como objetivo analizar las divergencias de las prácticas parentales, el comportamiento positivo y las problemáticas entre padres e hijos. Para esto, se establecieron dos objetivos específicos: (a) establecer la asociación entre las prácticas parentales y los problemas y conducta positiva de los adolescentes, tomando en cuenta las diadas padre-hijo, padre-hija, madre-hijo y madre-hija; y (b) identificar las diferencias entre las variables predictoras de las conductas en los adolescentes por medio de un análisis de senderos.

\section{Método}

\section{Tipo de estudio}

Se llevó a cabo un estudio cuantitativo, no experimental, con un diseño descriptivo y explicativo-correlacional para describir las variables del estudio y dar cuenta de su incidencia e interrelación (Hernández, Fernández \& Baptista, 2014).

\section{Participantes}

La muestra se seleccionó de manera no probabilística y por conveniencia en el sur de la Ciudad de México, y se compuso por diadas de padres e hijos organizadas de la siguiente manera: 248 padres-hijos, 241 padres-hijas, 237 madres-hijos, y 241 madres-hijas. Todos los participantes colaboraron de manera anónima y voluntaria tras la firma de un consentimiento informado. Con respecto a la edad, los hijos presentaron una media de 11.25 años $(D E=0.80)$, mientras que para las madres fue de 37.11 años $(D E=6.6)$ y para los padres, de 40.13 años $(D E=7.64)$.

\section{Instrumentos}

Escala de Capacidades y Dificultades (Goodman, 1997).

Este instrumento contiene seis factores: conducta prosocial, problemas de conducta, síntomas emocionales, hiperactividad/falta de atención, problemas con compañeros; y un factor de total de dificultades, donde se sumó el puntaje de los factores - a excepción de conducta prosocial-. Las opciones de respuesta para los niños fueron: "no es verdad", "es verdad a medias" y "verdaderamente sí"; mientras que las opciones de respuesta para las madres y los padres fueron: "no es cierto", "un tanto cierto" y "absolutamente cierto". El alfa total del instrumento fue de .727.

\section{Escala de Prácticas Parentales (Andrade \& Betancourt,} 2010).

Las dimensiones que evalúa esta escala son: (a) las prácticas parentales de la madre, que incluyen comunicación/ confianza, autonomía, imposición, control psicológico y control conductual; y (b) las prácticas parentales del padre, que incluyen comunicación/control conductual, autonomía, imposición y control psicológico. Las opciones de respuesta fueron "nunca", "pocas veces", "muchas veces" y "siempre". Se realizó una modificación en la redacción de los reactivos que no alteró los factores e índices de confiabilidad para que los hijos contestaran con respecto a su percepción de las prácticas parentales. En particular, la escala para las prácticas parentales de la madre presentó un alfa total de .821 , mientras que la de las prácticas parentales del padre fue de .895 .

\section{Procedimiento}

La aplicación de los instrumentos se realizó en dos fases, una dirigida a los adolescentes y otra a sus padres. En la 
primera fase, tras la obtención de la autorización para aplicar los cuestionarios a los adolescentes de escuelas de educación básica del sur de la Ciudad de México, se llevó a cabo la aplicación en los salones en horario escolar, con la presencia del profesor titular y de un aplicador capacitado para aclarar las dudas que surgían durante el proceso; se indicó a los participantes que sus respuestas serían anónimas y utilizadas con fines de investigación. Y en la segunda fase, se le envió a los padres un sobre con los cuestionarios y un consentimiento informado para colaborar en el estudio, donde se indicó que la aplicación sería anónima, con fines de investigación, y que no perjudicaría las evaluaciones escolares de sus hijos; se estableció un máximo de 3 días para entregar el sobre con los cuestionarios contestados al profesor. Al concluir el tiempo establecido, el aplicador acudió a las instituciones para recoger los cuestionarios. Las aplicaciones fueron realizadas con base en los estándares éticos del manual de la American Psychological Association (APA, 2010) y apegados a la Declaración de Helsinki (WMA, 1964).

\section{Análisis de datos}

Todos los análisis se realizaron mediante el programa estadístico IBM SPSS, v. 21, y AMOS, v. 21. Inicialmente, se realizó el análisis de correlación entre las prácticas parentales ejercidas tanto por la madre como por el padre con los problemas y la conducta positiva de los adolescentes; posteriormente, se llevó a cabo el análisis de senderos para observar la manera en que las prácticas parentales predicen el comportamiento de sus hijos; y, por último, se realizó un análisis de diferencias de medias de grupos relacionados (prueba t), con el objetivo de observar discrepancias en las prácticas parentales, los problemas y la conducta prosocial entre los padres y los adolescentes.

\section{Resultados}

En este apartado se presentan los resultados arrojados por cada uno de los análisis realizados, ordenados según el objetivo que perseguía cada uno de ellos.

\section{Correlaciones}

Los resultados obtenidos con respecto a la correlación $r$ de Pearson de las prácticas parentales de la madre y la conducta de los adolescentes se muestran en la Tabla 1, mientras que en la Tabla 2 aparecen los resultados de los padres; los resultados se organizaron según el sexo de los adolescentes. En las dos tablas se observan correlaciones significativas -de bajas a moderadas- en la mayoría de los factores. Es importante destacar que se obtuvieron puntuaciones diferentes cuando las prácticas parentales se dirigen hacia una mujer, ya que la correlación es significativa entre la conducta de la madre y su hija en el caso de la comunicación/confianza, síntomas emocionales e hiperactividad/falta de atención; asociación que no se encuentra cuando su hijo es varón. Sin embargo, la relación es inversa cuando hay problemas con compañeros.

Asimismo, en la Tabla 2 se pueden observar correlaciones significativas de bajas a moderadas en la mayoría de los factores. Al igual que en la tabla anterior, se observan algunas diferencias en cuanto al sexo de los hijos, ya que hay una correlación negativa entre la comunicación/control conductual y los síntomas emocionales cuando se trata de una hija, pero no se observa correlación cuando la práctica parental se dirige hacia un hijo. Además de esto, el control psicológico correlacionó con la conducta prosocial cuando el adolescente es hombre, pero no cuando es mujer.

Tabla 1.

Correlaciones entre las prácticas parentales de la madre y la conducta prosocial y problemas de los adolescentes

\begin{tabular}{|c|c|c|c|c|c|c|}
\hline Variables & & $\begin{array}{c}\text { Hiperactividad/Falta } \\
\text { de atención }\end{array}$ & $\begin{array}{c}\text { Síntomas } \\
\text { emocionales }\end{array}$ & $\begin{array}{c}\text { Problemas de } \\
\text { conducta }\end{array}$ & $\begin{array}{c}\text { Problemas con } \\
\text { compañeros }\end{array}$ & $\begin{array}{l}\text { Conducta } \\
\text { prosocial } \\
\end{array}$ \\
\hline \multirow[t]{2}{*}{ Comunicación/Confianza } & Hijos & -.104 & -.060 & $-.244 * *$ & $-.219 * *$ & $.330 * *$ \\
\hline & Hijas & $-.210 * *$ & $-.159 *$ & $-.237 * *$ & -.100 & $.322 * *$ \\
\hline \multirow[t]{2}{*}{ Autonomía } & Hijos & $-.132 * *$ & $-.176 * *$ & $-.308 * *$ & $-.280 * *$ & $.278 * *$ \\
\hline & Hijas & $-.272 * *$ & $-.269 * *$ & $-.347 * *$ & $-.187 * *$ & $.289 * *$ \\
\hline \multirow[t]{2}{*}{ Imposición } & Hijos & $.192 * *$ & $.260 * *$ & $.291 * *$ & $.153 *$ & .045 \\
\hline & Hijas & $.175^{* *}$ & $.260 * *$ & $.278 * *$ & $.183 * *$ & -.089 \\
\hline \multirow[t]{2}{*}{ Control psicológico } & Hijos & $.136^{*}$ & $.178 * *$ & $.292 * *$ & $.225 * *$ & -.010 \\
\hline & Hijas & $.220 * *$ & $.258 * *$ & $.361 * *$ & $.163 *$ & $-.196 * *$ \\
\hline \multirow[t]{2}{*}{ Control conductual } & Hijos & $-.144 * *$ & -.071 & $-.223 * *$ & $-.301 * *$ & $.380 * *$ \\
\hline & Hijas & $-.302 * *$ & $-.209 * *$ & $-.332 * *$ & $-.127 *$ & $.335 * *$ \\
\hline
\end{tabular}

Nota. $* *$ Correlación significativa a $.01 ; *$ Correlación significativa a .05 . 
Tabla 2.

Correlación entre las prácticas parentales del padre y los sintomas emocionales y problemas de conducta de las adolescentes

\begin{tabular}{|c|c|c|c|c|c|c|}
\hline Variables & & $\begin{array}{l}\text { Hiperactividad/ } \\
\text { Falta de atención }\end{array}$ & $\begin{array}{c}\text { Síntomas emo- } \\
\text { cionales }\end{array}$ & $\begin{array}{c}\text { Problemas de } \\
\text { conducta }\end{array}$ & $\begin{array}{c}\text { Problemas con } \\
\text { compañeros }\end{array}$ & $\begin{array}{l}\text { Conducta } \\
\text { prosocial }\end{array}$ \\
\hline \multirow[t]{2}{*}{ Comunicación/Control conductual } & Hijos & $-.152 * *$ & -.090 & $-.166^{* *}$ & $-.268^{*}$ & $.325 * *$ \\
\hline & Hijas & $-.282 * *$ & $-.278 * *$ & $-.260 * *$ & $-.139 *$ & $.225 * *$ \\
\hline \multirow[t]{2}{*}{ Autonomía } & Hijos & $.274 * *$ & $-.186 * *$ & $-.247 * *$ & $-.313 * *$ & $.307 * *$ \\
\hline & Hijas & $-.289 * *$ & $-.214 * *$ & $-.313 * *$ & $-.142 *$ & $.295 * *$ \\
\hline \multirow[t]{2}{*}{ Imposición } & Hijos & $.249 * *$ & $.239 * *$ & $.195 * *$ & $.165 * *$ & .080 \\
\hline & Hijas & $-.125 * *$ & $.224 * *$ & $.167 * *$ & $.126 * *$ & -.013 \\
\hline \multirow[t]{2}{*}{ Control psicológico } & Hijos & $.265 * *$ & $.270 * *$ & $.221 * *$ & $.204 * *$ & $-.125 * *$ \\
\hline & Hijas & $.199 * *$ & $.278 * *$ & $.316^{* *}$ & $.207 * *$ & .083 \\
\hline
\end{tabular}

Nota. ** Correlación significativa a $.01 ; *$ Correlación significativa a .05 .

Modelos de análisis de senderos

Por lo que refiere a las diferencias entre las variables predictoras de las conductas de los adolescentes, se realizó un análisis de senderos teniendo en cuenta los criterios del índice de ajuste de chi-cuadrado ajustado $\left(x^{2}\right)$ y ajuste normado de Bentler-Bonett (NFI), a partir de los cuales se compara el modelo propuesto y el modelo nulo, donde un valor es aceptable si es mayor a .90. Asimismo, se retomó el índice de ajuste de incremento de Bollet (IFI), el índice comparativo de ajuste de Bentler (CFI) y el error cuadrático medio de aproximación (RMSEA), de acuerdo con Manzano y Zamora (2009), con un punto de corte aceptable si el ajuste es de .90. Para el RMSEA se recomienda que el ajuste sea menor a .05 , ya que si es menor o igual a .05 , significa un error de aproximación del modelo con la realidad. Los modelos obtenidos en este estudio cumplieron ajustes aceptables.
Para la realización de los modelos se tomó en cuenta el sexo de los hijos. Específicamente, en el modelo de madres e hijas se obtuvieron los siguiente índices de ajuste: $\chi^{2}=18.546, \mathrm{gl}=11(\mathrm{p}>.05), \mathrm{CFI}=.994, \mathrm{NFI}=.987$, $\mathrm{IFI}=.994$ y RMSEA $=.035(\mathrm{LO} 90=.000$ y HI $90=.062$; mientras que para el modelo de madres e hijos se obtuvieron: $\chi^{2}=13.266, \mathrm{gl}=10(\mathrm{p}>.05), \mathrm{CFI}=.997, \mathrm{NFI}=.990$, IFI $=.998$ y RMSEA $=.024($ LO $90=.000$ y HI $90=.55)$.

De acuerdo con los datos obtenidos en los modelos de análisis de senderos, se encontraron variantes del impacto de las prácticas parentales de la madre en el comportamiento de sus hijos e hijas, tal como se observa en la Tabla 3. Para los hijos, la práctica parental que más se repite fue la de autonomía, seguida de control psicológico, control conductual, comunicación e imposición; mientras que para las hijas la conducta más frecuente fue la de autonomía, seguida de control psicológico, comunicación, control conductual e imposición.

Tabla 3.

Predicción de las prácticas parentales de la madre en la conducta prosocial y los problemas de los adolescentes

\begin{tabular}{|c|c|c|c|c|c|c|}
\hline \multicolumn{2}{|c|}{ Hijas } & $\beta$ & $r^{2}$ & Hijos & $\beta$ & $\mathrm{r}^{2}$ \\
\hline \multirow{4}{*}{ Conducta prosocial } & Comunicación & .13 & .10 & Comunicación & .17 & .15 \\
\hline & Autonomía & .15 & & Autonomía & .12 & \\
\hline & Control conductual & .11 & & Imposición & .09 & \\
\hline & & & & Control conductual & .20 & \\
\hline \multirow{3}{*}{$\begin{array}{l}\text { Hiperactividad/Falta de } \\
\text { atención }\end{array}$} & Autonomía & .17 & .12 & Comunicación & -.11 & .09 \\
\hline & Control conductual & -.12 & & Autonomía & -.13 & \\
\hline & Control psicológico & .18 & & Control psicológico & .17 & \\
\hline \multirow{3}{*}{ Síntomas emocionales } & Autonomía & -.18 & .12 & Autonomía & -.22 & .11 \\
\hline & Control psicológico & .14 & & Control psicológico & .19 & \\
\hline & Control conductual & -.15 & & & & \\
\hline \multirow{3}{*}{ Problemas de conducta } & Autonomía & -.16 & .14 & Comunicación & -.14 & .15 \\
\hline & Control conductual & .09 & & Autonomía & -.15 & \\
\hline & Control psicológico & .25 & & Control psicológico & .22 & \\
\hline \multirow{3}{*}{$\begin{array}{l}\text { Problemas con } \\
\text { compañeros }\end{array}$} & Autonomía & -.13 & .05 & Autonomía & -.12 & .11 \\
\hline & Control psicológico & .16 & & Control psicológico & .17 & \\
\hline & & & & Control conductual & -.18 & \\
\hline
\end{tabular}


Por otra parte, se obtuvieron dos modelos que cumplieron con los índices de ajuste adecuado para la explicación de las prácticas parentales de los padres y las respuestas de sus hijos, donde el modelo de padres e hijas obtuvo los ajustes: $\chi^{2}=16.652, \mathrm{gl}=11(\mathrm{p}>.05), \mathrm{CFI}=.994$, $\mathrm{NFI}=.984$, IFI $=.995$ y RMSEA $=.032($ LO $90=.000$ y HI $90=.062)$; mientras que para el modelo de padres $e$ hijos fueron: $\chi^{2}=32.660, \mathrm{gl}=13(\mathrm{p}<.05), \mathrm{CFI}=.981$, $\mathrm{NFI}=.969, \mathrm{IFI}=.981$ y RMSEA $=.055(\mathrm{LO} 90=.32 \mathrm{y}$ HI $90=.79$ ).

Estos resultados se muestran en la Tabla 4, e indican que la práctica que más impacta en el comportamiento de los hijos fue el control conductual/comunicación, seguida de autonomía, control psicológico e imposición; mientras que para las hijas fue el control conductual/comunicación, seguido de autonomía, control psicológico e imposición.

\section{Análisis de comparación de grupos}

Para los análisis de comparación de grupos se tomaron diadas con las respuestas tanto de los padres y madres como de sus hijos e hijas, y se ejecutó un análisis de prueba $\mathrm{t}$ con muestras relacionadas. Cabe mencionar que se cubrió el criterio de normalidad mediante los índices de asimetría y curtosis (los resultados se aprecian en la Figura 1). Específicamente, se encontró que en las prácticas parentales que ejercen los padres hubo diferencias significativas en los factores de comunicación/control conductual ( $t=2.011$, sig. $<.05 ; t=3.022$, sig. $<.05)$ y control psicológico $(t=2.618$, sig. $<.05 ; t=2.175$ sig. $<.05)$, siendo los padres quienes consideran que ponen en práctica estas conductas parentales en comparación con la percepción de sus hijos e hijas. Además, en el caso de las hijas también se presentó la conducta de imposición $(t=1.988$, sig. $<.05)$, siendo nuevamente los padres quienes perciben más esta práctica que sus hijas.

Los resultados obtenidos con respecto a la percepción entre madres y sus hijos se pueden observar en la Figura 2. En particular, se encontró que en las prácticas parentales de comunicación $(\mathrm{t}=5.655$, sig. $<.05)$, autonomía $(t=2.183$, sig. $<.05)$, control psicológico $(t=2.593$, sig. $<.05)$ y control conductual $(t=8.216$, sig. $<.05)$, había diferencias significativas, ya que las madres perciben más esta conducta que sus hijos. Mientras que, en el caso de las hijas, solo se identificaron diferencias en imposición $(t=3.569$, sig. $<.05)$, control psicológico $(t=4.389$, sig. $<.05) \mathrm{y}$ control conductual $(t=5.052$, sig. $<.05)$, ya que, al igual que en el caso anterior, las madres mencionan ejercer más esta práctica que lo percibido por sus hijas.

En la Figura 3 se muestran los datos obtenidos en el análisis de prueba t con muestras relacionadas, donde se obtuvo que, en el caso de los padres, sus hijos e hijas perciben más sintomas emocionales $(t=-4.116$, sig. $<.05 ; t=-8.022$, sig. $<.05)$, problemas con compañeros $(t=-6.217$, sig. $<.05$; $t=-2.696$, sig. $<.05)$ y total de dificultades $(t=-5.514$, sig. $<.05 ; t=-3.272$, sig. $<.05)$, en comparación con sus padres. En el caso de los hijos, se reportó más percepción de hiperactividad $(t=3.919$, sig. $<.05)$, mientras que, para las hijas, más conducta prosocial $(t=-3.021$, sig. $<.05)$.

Tabla 4.

Predicción de las prácticas parentales del padre en la conducta prosocial y problemas de los adolescentes

\begin{tabular}{|c|c|c|c|c|c|c|}
\hline & Hijas & $\beta$ & $\mathrm{r}^{2}$ & Hijos & $\beta$ & $\mathrm{r}^{2}$ \\
\hline \multirow{3}{*}{$\begin{array}{l}\text { Conducta } \\
\text { prosocial }\end{array}$} & $\begin{array}{l}\text { Control conductual/ } \\
\text { Comunicación }\end{array}$ & .12 & \multirow[t]{3}{*}{.07} & $\begin{array}{l}\text { Control Conductual/ } \\
\text { Comunicación }\end{array}$ & .21 & \multirow[t]{3}{*}{.12} \\
\hline & Autonomía & .19 & & Autonomía & .18 & \\
\hline & & & & Imposición & .10 & \\
\hline \multirow{2}{*}{$\begin{array}{l}\text { Hiperactividad/ } \\
\text { Falta de atención }\end{array}$} & $\begin{array}{l}\text { Control conductual/ } \\
\text { Comunicación }\end{array}$ & -.16 & \multirow[t]{2}{*}{.11} & $\begin{array}{l}\text { Control conductual/ } \\
\text { Comunicación }\end{array}$ & -.13 & \multirow[t]{2}{*}{.09} \\
\hline & Autonomía & -.21 & & Autonomía & -.21 & \\
\hline \multirow{2}{*}{$\begin{array}{l}\text { Síntomas } \\
\text { Emocionales }\end{array}$} & $\begin{array}{l}\text { Control conductual/ } \\
\text { Comunicación }\end{array}$ & -.25 & \multirow[t]{2}{*}{.10} & $\begin{array}{l}\text { Control conductual/ } \\
\text { Comunicación }\end{array}$ & -.16 & \multirow[t]{2}{*}{.07} \\
\hline & Control psicológico & .13 & & Control psicológico & .16 & \\
\hline \multirow{3}{*}{$\begin{array}{l}\text { Problemas de } \\
\text { Conducta }\end{array}$} & $\begin{array}{l}\text { Control conductual/ } \\
\text { Comunicación }\end{array}$ & -.11 & \multirow[t]{3}{*}{.13} & $\begin{array}{l}\text { Control conductual/ } \\
\text { Comunicación }\end{array}$ & -.17 & \multirow[t]{3}{*}{.08} \\
\hline & Autonomía & -.18 & & Autonomía & -.16 & \\
\hline & Control psicológico & .16 & & & & \\
\hline \multirow{2}{*}{$\begin{array}{l}\text { Problemas con } \\
\text { compañeros }\end{array}$} & Autonomía & -.13 & \multirow[t]{2}{*}{.05} & $\begin{array}{l}\text { Control conductual/ } \\
\text { Comunicación }\end{array}$ & -.19 & \multirow[t]{2}{*}{.09} \\
\hline & Control psicológico & .14 & & Autonomía & -.19 & \\
\hline
\end{tabular}




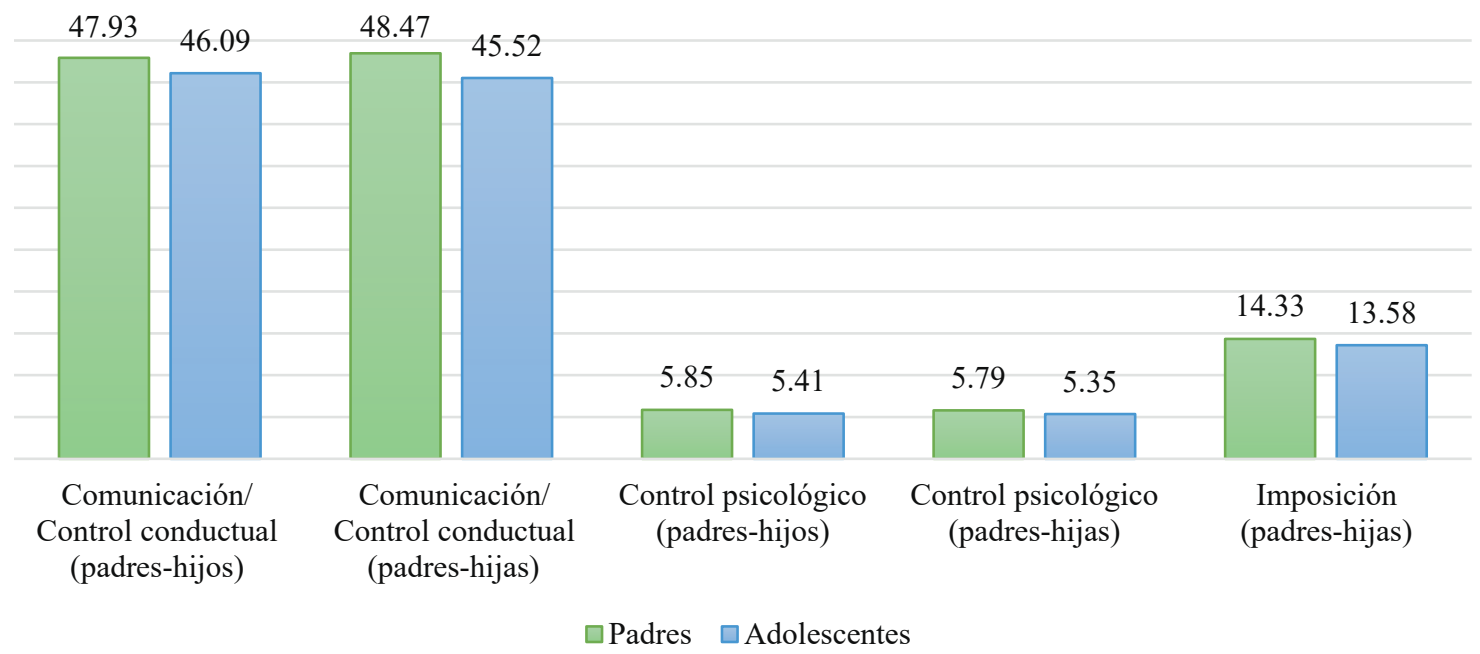

Figura 1. Diferencia de medias entre las prácticas parentales de los padres y sus hijos e hijas.

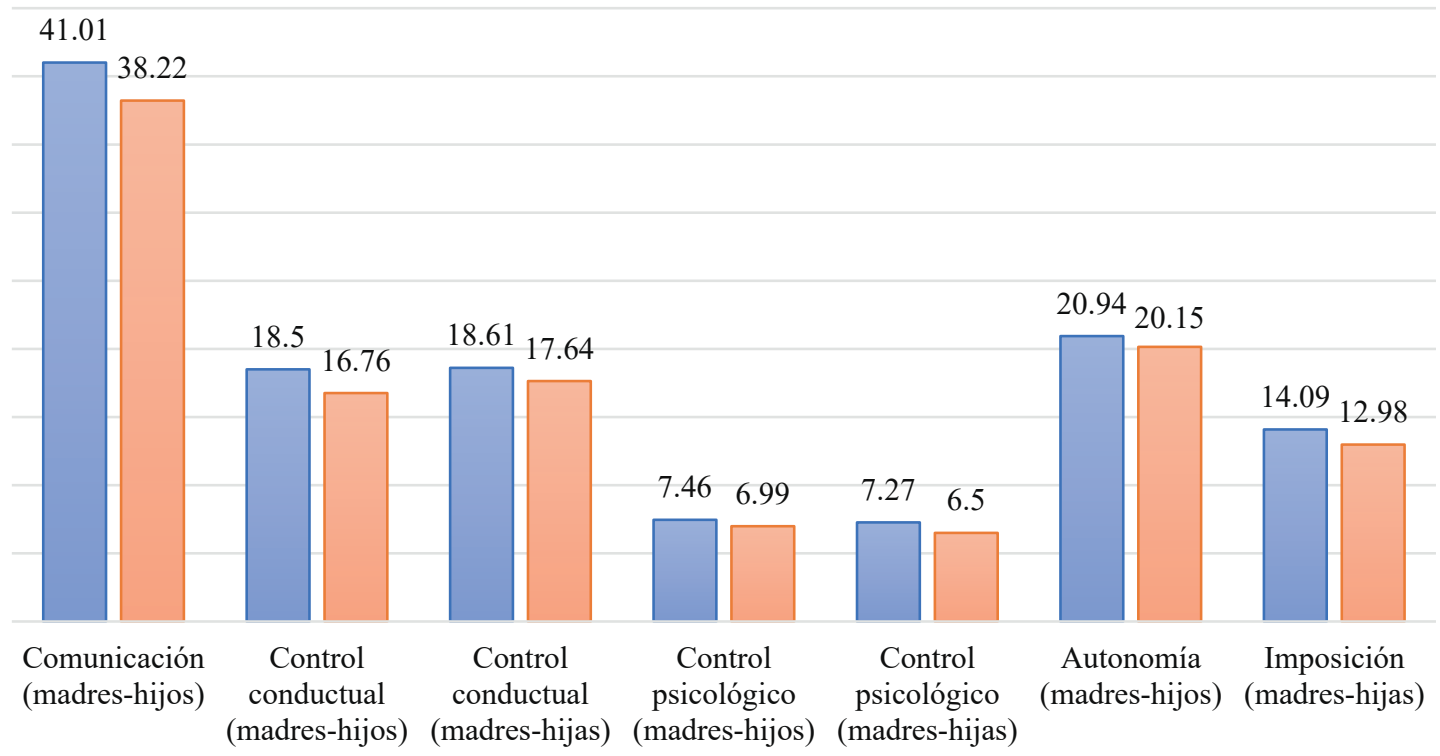

$\square$ Madres $\square$ Adolescentes

Figura 2. Diferencia de medias entre las prácticas parentales de las madres y sus hijos e hijas.

Por último, se obtuvieron datos similares en la percepción de la madre y sus hijos (véase Figura 4), ya que se encontraron diferencias en sintomas emocionales $(t=-2.288$, sig. $<.05)$, problemas de conducta $(t=-2.235$, sig. $<.05)$, problemas con compañeros $(t=-3.634$, sig. $<.05)$ y total de dificultades $(t=-3.575$, sig. $<.05)$, debido a que los hijos reportaron más estos problemas en comparación con lo percibido por sus madres. Sin embargo, se encuentra lo inverso cuando se trata de la conducta prosocial, ya que las madres reportaron más esta conducta $(t=2.161$, sig. < .05) que los hijos. En el caso de las diadas madrehija, solo se encontraron diferencias en dos factores: el de problemas emocionales $(t=-5.423$, sig. $<.05)$, en donde las hijas reportan más estos problemas; y el de problemas de conducta $(t=2.437$, sig. $<.05)$, ya que las madres perciben más esta problemática que sus hijas.

\section{Discusión}

El objetivo principal de la presente investigación fue analizar las divergencias en la percepción de las prácticas parentales, el comportamiento positivo y las problemáticas entre padres e hijos, a partir del cual se establecieron dos 


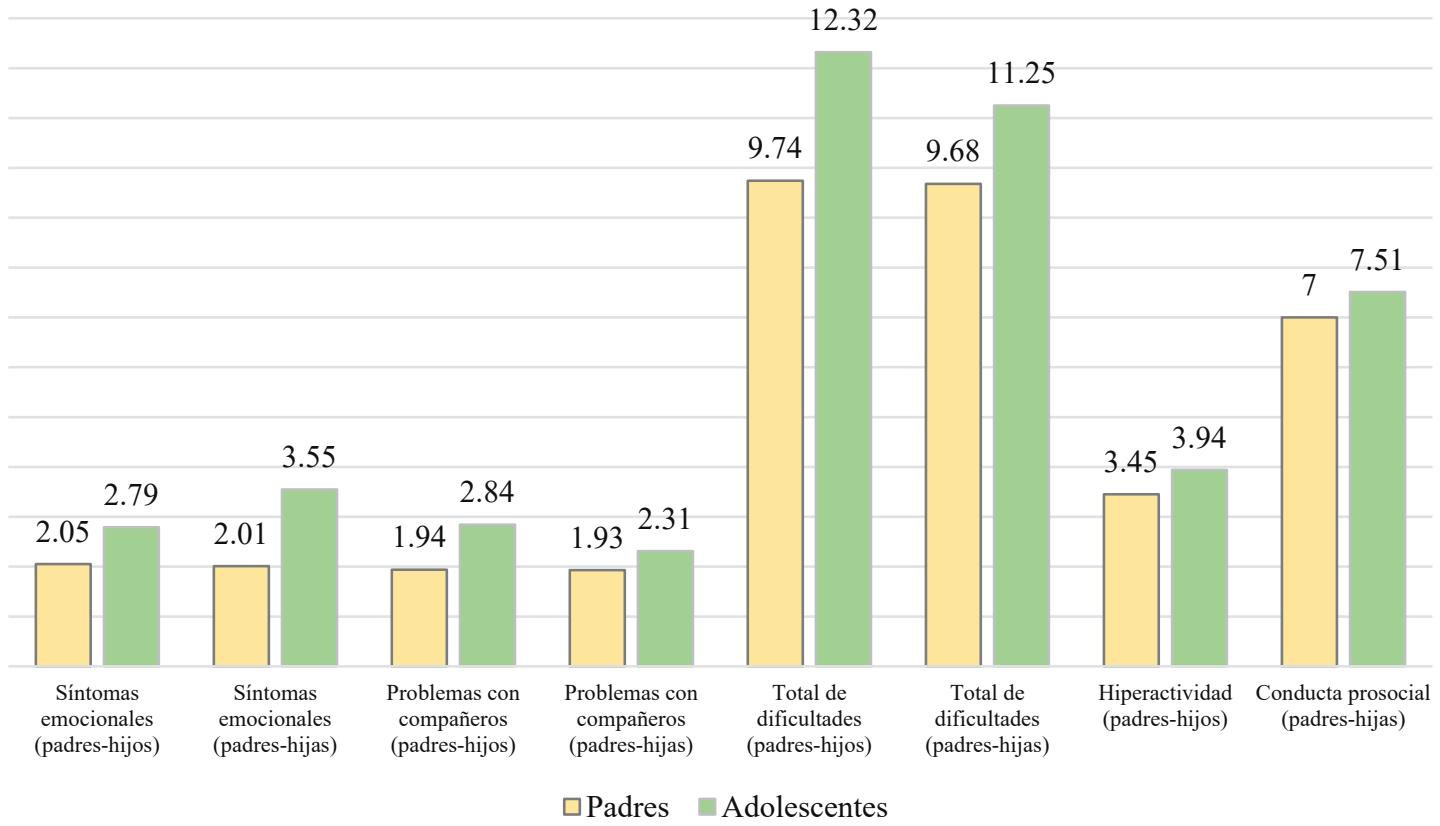

Figura 3. Diferencia de medias entre la conducta prosocial y los problemas de acuerdo con los reportes de los padres y sus hijos e hijas.

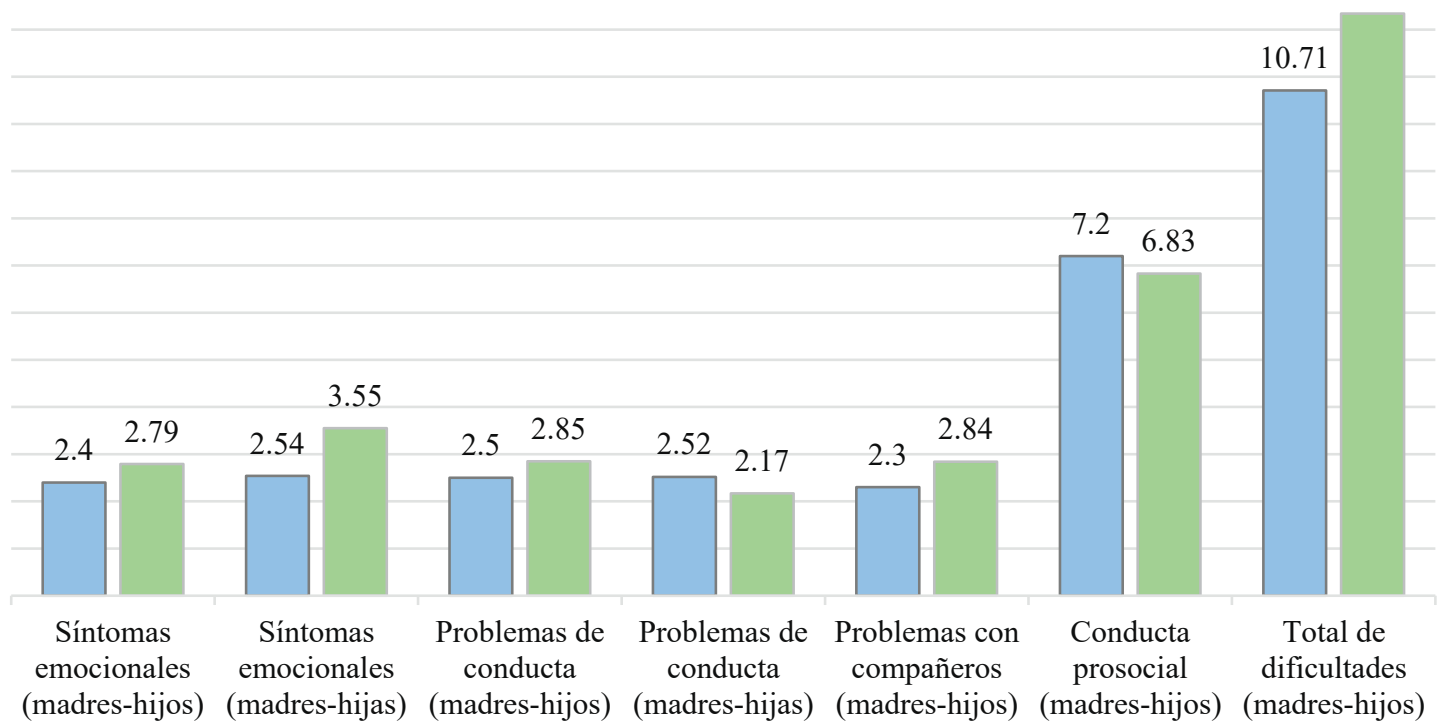

$\square$ Madres Adolescentes

Figura 4. Diferencia de medias entre la conducta prosocial y los problemas de acuerdo con los reportes de las madres y sus hijos e hijas.

objetivos específicos: analizar la asociación entre las prácticas parentales con los problemas y la conducta positiva de los adolescentes, y dar cuenta de las diferencias entre las variables predictoras de las conductas en los adolescentes por medio de un análisis de senderos.
En los resultados, los datos obtenidos del análisis entre la asociación de las prácticas parentales y los problemas y la conducta positiva de los adolescentes concuerdan con los estudios de Álvarez (2010), Betancourt y Andrade (2011) y Coln et al. (2013), debido a que se encontraron relaciones positivas entre las conductas parentales y los problemas 
en adolescentes, es decir, que las conductas parentales negativas -como imposición y control psicológico- se asociaron con hiperactividad/falta de atención, problemas de conducta, problemas con compañeros y síntomas emocionales; mientras que, por el contrario, la comunicación/ confianza y el fomento de la autonomía se relacionaron de manera negativa con los problemas en los adolescentes y de manera positiva con la conducta prosocial, la cual, como lo reportó Richaud de Minzi et al. (2011), disminuye ante el desinterés parental, la negligencia o el control patológico. Por lo tanto, es importante que la educación parental se brinde mediante prácticas positivas -como la comunicación, la autonomía y el control conductual- con el fin de disminuir problemáticas en los hijos e incrementar conductas positivas -como la conducta prosocial-.

Con respecto a los análisis de senderos, en el presente estudio se encontraron diferencias en las variables predictoras de las conductas parentales en los adolescentes, ya que, en cuanto a las madres, se presentó con mayor frecuencia comunicación, autonomía y control psicológico, mientras en los padres se presentó mayor control psicológico y control conductual. Estos resultados se asemejan con los reportado por Oudhof et al. (2012), donde se menciona que las madres pasan mayor tiempo con sus hijos y están más pendientes de sus actividades, lo que podría aumentar las conductas parentales de comunicación y control conductual, a diferencia de los padres, quienes reportan más control y no se percatan de las actividades de sus hijos (Rodrigo et al., 2005).

De esta forma, los modelos presentados confirman lo mencionado por Rocha (2004) sobre la deseabilidad social y los diferentes roles que adoptan los padres dependiendo del sexo de los hijos, sobre todo en lo relacionado con el distanciamiento del padre, ya que estos obtuvieron valores menores con respecto a las madres tanto en las correlaciones como en los diferentes valores de $\mathrm{r}^{2}$ de los modelos de senderos. Esto implica el juego de roles tradicionalista donde los padres se involucran de menor manera en la crianza de los hijos, a la vez que se comunican menos con ellos (Rodríguez et al., 2009; Sevilla et al., 2016).

Adicionalmente, en el objetivo principal se planteó analizar las divergencias en la percepción de las prácticas parentales, el comportamiento positivo y las problemáticas entre padres e hijos, y en los resultados se obtuvieron diferencias de puntajes entre padres e hijos tanto en las prácticas parentales como en los problemas y la conducta prosocial. En particular, en cuanto a las prácticas parentales, los padres demostraron tener una percepción de que controlan a sus hijos conductual y psicológicamente mucho mayor a la que reportaron los hijos e hijas, es decir, que creen que conocen y, por tanto, saben en dónde y qué hacen sus hijos, pero los hijos no perciben que este control sea de la misma magnitud. Además, los padres piensan que ejercen cierto tipo de control psicológico sobre sus hijos, esto es, que los chantajean y los llegan a amenazar, pero sus hijos e hijas no creen lo mismo y puntúan en menor medida. En el caso de las madres, hubo una diferencia similar a la de los padres tanto en hijos como en hijas, ya que, de la misma manera, perciben que controlan la conducta de sus hijos en una magnitud mayor a lo que los adolescentes reportaron.

Por otra parte, en las madres se encontraron variaciones en las prácticas parentales según el sexo de los hijos, ya que, por ejemplo, en el caso de los hombres, las madres reportaron una mayor comunicación con ellos, mientras que con las mujeres piensan que les imponen y que las chantajean o amenazan más de lo que sus hijas reportan. Esto implica que la madre tiende a comportarse de manera diferente dependiendo del sexo de su hijo.

Incluso, madres y padres tienden a subestimar los problemas que sus hijos e hijas tienen, ya que, según lo que demuestran los datos encontrados en este estudio, los padres indicaron menores niveles de problemas de conducta, síntomas emocionales y problemas con compañeros que los reportados sus hijos; además de que desfavorecen la conducta prosocial de sus hijas y reportan menores puntajes que ellas en cuanto a sus dificultades.

Paradójicamente, las madres reportan una mayor cantidad de problemas de conducta en sus hijas comparado con los puntajes de estas y sobrestiman la conducta prosocial de los hijos. Lo anterior pone de manifiesto una tendencia preocupante hacia los estereotipos de género, donde el hombre no tiene problemas y la mujer puede incluso ser percibida con menos problemas de conducta que los que en realidad presenta. Esto concuerda con lo reportado por autores como Aguilar-Morales et al. (2006), donde se menciona que algunos padres reportan conductas consideradas como problemáticas, y que gran parte de los diagnósticos se establecen por las complicaciones que genera la conducta de los hijos en los padres y no por lo reportado por los hijos mismos.

Teniendo esto en cuenta, se puede concluir que existen divergencias entre los reportes de prácticas parentales, la conducta prosocial y los problemas entre los padres y sus hijos, lo cual implica que los padres muchas veces no tienen un conocimiento directo de lo que hacen sus hijos y que no ejercen adecuadas prácticas parentales a pesar de estar convencidos de que sí lo hacen. De igual forma, la influencia cultural es innegable al ser los hombres considerados tanto por padres como por madres como con menos problemas y con mayor conducta prosocial; y, en cambio, las mujeres son consideradas, por lo menos por la madre, como con más problemas. Aunado a este efecto del género, se encontró 
que la madre sigue teniendo un papel central en la crianza de los hijos de cualquier sexo, ya que por medio de sus prácticas parentales tiene más influencia en el desarrollo de problemas y en la conducta prosocial de sus hijos.

Por último, es importante mencionar que algunas de las limitaciones del estudio fueron la muestra no probabilística, ya que no es representativa de la población mexicana; que la validez interna y externa se vio afectada debido a que no se pudieron controlar los factores ambientales, organísmicos y situacionales en la aplicación; y que, con respecto al análisis de datos y el error de medida, se recomienda replicar el estudio con una muestra representativa más grande con el fin de disminuir este tipo de error.

En conclusión, en el presente trabajo se puede observar la necesidad de diseñar estrategias de intervención en donde se fomenten prácticas parentales positivas que disminuyan los problemas que presentan los adolescentes y que, por medio de la comunicación, los padres conozcan las actividades de sus hijos y los apoyen cuando lo necesiten. Con esto será posible disminuir las discrepancias en la percepción de las prácticas parentales, los problemas y la conducta positiva entre padres e hijos.

\section{Referencias}

Aguilar-Morales, E. O., Aguilar-Morales, J. E., \& VargasMendoza, J. E. (2006). Problemas de conducta infantil en una comunidad de la mixteca oaxaqueña. Centro Regional de Investigación en Psicología, 1(1), 29-32.

Álvarez, G. M. (2010). Prácticas educativas parentales: autoridad familiar, incidencia en el comportamiento agresivo infantil. Revista Virtual Universidad Católica del Norte, 1(31), 253-273. Recuperado de http://revistavirtual.ucn. edu.co/index.php/RevistaUCN/article/viewFile/44/97

Andrade, P. P., \& Betancourt, O. D. (2010). Evaluación de las prácticas parentales en padres e hijos. En A. S. Rivera, R. Díaz-Loving, L., I. Reyes, A. R. Sánchez \& M. L. M. Cruz (Eds.), La psicología social en México (vol. XIII, pp. 137142). México: AMEPSO.

American Psychological Association. (2010). Manual de Publicaciones de la APA. México: El Manual Moderno.

Bailey, M. K., Zauszniewski, J. A., Heinzer, M. M., \& Hemstrom-Krainess (2007). Patterns of Depressive Symptoms in Children. Journal of Child Adolescent Psychiatric Nursing, 20(2), 86-95. doi: https://doi. org/10.1111/j.1744-6171.2007.00090.x

Betancourt, O. D., \& Andrade, P. P. (2011). Control parental y problemas emocionales y de conducta en adolescentes. Revista Colombiana de Psicología, 20(1), 2741. Recuperado de https://dialnet.unirioja.es/servlet/ articulo? codigo $=4035676$
Campos, M., Eceiza, A, \& Páez, D. (2004). Socialización, familia y cultura. En D. Páez, I. Fernández, S. Ubillos \& E. Zubieta (Ed.), Psicología Social, Cultura y Educación (pp. 845-888). España: Pearson Prentice Hall.

Castro, F. J. (2005). Disciplina y estilo educativo familiar. En L. Ezpeleta, Factores de riesgo en psicopatología del desarrollo (pp. 319-336). España: Masson.

Coln, K. L., Jordan, S. S., \& Mercer, S. H. (2013). A unified model exploring parenting practices as mediators of marital conflict and children's adjustment. Child Psychiatry \& Human Development, 44(3), 419-429. doi: https://doi. org/10.1007/s10578-012-0336-8

Darling, N., \& Steinberg, L. (1993). Parenting style as context: An Integrative model. Psychological Bulletin, 113(3), 487496. doi: http://dx.doi.org/10.1037/0033-2909.113.3.487

Davé, S., Sherr, L., Senior, R., \& Nazareth, I. (2008). Associations between paternal depression and behavior problems in children of 4-6 years. European Child Adolescent Psychiatry, 17, 306-315. doi: https://doi.org/10.1007/ s00787-007-0672-6

De La Peña Olvera, F. R., Gómez C. C., Heinze M., G., \& Palacios-Cruz, L. (2014). Adversidad social y trastornos psiquiátricos: Estudio comparativo entre estudiantes de secundarias públicas y privadas. Salud mental, 37(6), 483489. Recuperado de https://www.medigraphic.com/pdfs/ salmen/sam-2014/sam146f.pdf

Díaz, R. C., \& González, R. M. (2014). Conductas problema en adolescentes en la ciudad de Monterrey, México. Enfermería Global, 33(1), 1-16. doi: https://doi. org/10.6018/eglobal.13.1.184861

Elgar, F. J., Mills, R. S., McGrath, P. J., Waschbusch, D. A., \& Brownridge, D. A. (2007). Maternal and Paternal Depressive Symptoms and Child Maladjustment: The Mediating Role of Parental Behavior. Journal Abnormal Child Psychology, 35(6), 943-955. doi: https://doi. org/10.1007/s10802-007-9145-0

Giannakopoulos, G., Tzavara, C., Dimitrakaki, C., Kolaitis, G., Rotsika, V., \& Tountas Y. (2009). The factor structure of the Strengths and Difficulties Questionnaire (SDQ) in Greek adolescents. Annals of General Psychiatry, 20(8), 1-7. doi: https://doi.org/10.1186/1744-859X-8-20

Gómez-Ortiz, O., Del Rey, R., Romera, E. M., \& Ortega-Ruiz, R. (2015). Los estilos educativos paternos y maternos en la adolescencia y su relación con la resiliencia, el apego y la implicación en acoso escolar. Anales de Psicología, 31(3), 979-989. doi: https://doi.org/10.6018/analesps.31.3.180791

Goodman, R. (1997). The Strengths and Difficulties Questionnaire: A Research Note. Journal Child Psychology and Psychiatry, 38(5), 581-587. doi: https://doi. org/10.1111/j.1469-7610.1997.tb01545.x

Hall, L. A., Rayens, M. K., \& Peden A. R. (2008). Maternal Factors Associated with Child Behavior. Journal of Nursing Scholarship, 40(2), 124-130. doi: https://doi. org/10.1111/j.1547-5069.2008.00216.x 
Hernández, N. J., Guadarrama, G. R., Veytia, L. M., \& Márquez, M. O. (2017). Prevalencia de problemas emocionales y conductuales en una muestra de adolescentes mexicanos. Psico, 48(4), 250-255. doi: http://dx.doi. org/10.15448/1980-8623.2017.4.25438

Hernández, S. R., Fernández, C. C., \& Baptista, L. P. (2014). Metodología de la investigación (6. ${ }^{\mathrm{a}}$ ed.). México D. F.: McGraw-Hill.

Instituto Nacional de Estadística y Geografía (INEGI) (2015). Encuesta Intercensal 2015. Recuperado de http://www.beta. inegi.org.mx/temas/estructura/

Jiménez-Iglesias, A., \& Moreno C. (2015). La influencia de las diferencias entre el padre y la madre sobre el ajuste adolescente. Anales de psicología, 31(1), 367-377. doi: http:// dx.doi.org/10.6018/analesps.31.1.158081

Manzano, P. A., \& Zamora, M. S. (2009). Sistema de ecuaciones estructurales: una herramienta de investigación (Cuaderno técnico 4). México: Centro Nacional de Evaluación para la Educación Superior, A. C.

Meier, L. K., \& Oros, L. B. (2012). Percepción de las prácticas parentales y experiencia de emociones positivas en adolescentes, Revista de Psicología, 8(16), 73-84. Recuperado de http://hdl.handle.net/11336/19108

Méndez, S. M., Andrade, P. P., \& Peñaloza, G. R. (2013). Prácticas parentales y capacidades y dificultades en preadolescentes. Revista Intercontinental de Psicología y Educación, 15(1), 99-118. Recuperado de https://www.redalyc.org/html/802/80225697007/

Morales, C. S., Félix, R. V., Rosas, P. M., López, C. F., \& Nieto, G. J. (2015). Prácticas de Crianza Asociadas al Comportamiento Negativista Desafiante y de Agresión Infantil. Avances en Psicología Latinoamericana 33(1), 5776. doi: dx.doi.org/10.12804/ap133.01.2015.05

Oliva, D. A., Parra, J. A., Sánchez-Queija, I., \& López, G. F. (2007). Estilos educativos materno y paterno: evaluación y relación con el ajuste adolescente. Anales de la Psicología, 23(1), 49-56. Recuperado de https://revistas.um.es/ analesps/article/view/23201

Organización Mundial de la Salud(OMS) (2018). Salud de la madre, el recién nacido, del niño y del adolescente. Recuperado de http://www.who.int/maternal_child_adolescent/topics/ adolescence/dev/es/

Oudhof, B. H., Rodríguez, A. B., \& Robles, E. E. (2012). La percepción de la crianza en padres, madres e hijos adolescents. Liberabit, 18(1), 75-82. Recuperado de http:// www.scielo.org.pe/scielo.php?pid=S1729-482720120 00100010\&script $=$ sci_arttext

Papalia, D. E., \& Martorell, G. (2017). Desarrollo humano (13. ed.). México: McGraw-Hill Education.

Parra, A., \& Oliva A. (2002). Comunicación y conflicto familiar durante la adolescencia. Anales de Psicología, 18(2),
215-223. Recuperado de https://revistas.um.es/analesps/ article/view/28421

Reising, M. M., Watson, K. H., Hardcastle, E. J., Merchant, M. J., Roberts, L., Forehand, R., \& Compas, B. E. (2013). Parental depression and economic disadvantage: The role of parenting in associations with internalizing and externalizing symptoms in children and adolescents. Journal of child and family studies, 22(3), 335-343. doi: https://doi. org/10.1007/s10826-012-9582-4

Richaud de Minzi, M.C., Lemos, V., \& Mesurado B. (2011). Relaciones entre la percepción que tienen los niños de los estilos de relación y de la empatía de los padres y la conducta prosocial en la niñez media y tardía. Avances en Psicología Latinoamericana, 29(2), 330-343. Recuperado de https://revistas.urosario.edu.co/index.php/apl/article/ view/1363

Rocha, S. T. (2004). Socialización, cultura e identidad de sexo: el impacto de la diferenciación entre los sexos (tesis de doctorado). Facultad de Psicología. UNAM.

Rodrigo, J. M., García, M., Máiquez M. L., \& Triana, B. (2005). Discrepancias entre padres e hijos adolescentes en la frecuencia percibida e intensidad emocional en los conflictos familiars. Estudios de Psicología, 26(1), 21-34. doi: http:// dx.doi.org/10.1174/0210939053421407

Rodríguez, M. A., Del Barrio, M. V., \& Carrasco M. A. (2009). ¿Cómo perciben los hijos la crianza maternal y paterna? Diferencias por edad y sexo. Escritos de Psicología, 2(2), 10-18. Recuperado de http://scielo.isciii.es/pdf/ep/v2n2/ original2.pdf

Schojaei, T., Wazana, A., Pitrou, I., \& Kovess, V. (2009). The strengths and difficulties questionnaire: validation study in French school-aged child8ren and cross-cultural comparisons. Social Psychiatry and Psychiatric Epidemiology, 44(9), 740-747. doi: https://doi.org/10.1007/ s00127-008-0489-8

Sevilla, T. M., Sanabria, J. P., Orcasita, L. T., \& Palma, D. M. (2016). Consistencies and discrepancies in communication between parents and teenage children about sexuality. Paidéia (Ribeirão Preto), 26(64), 139-147. doi: 10.1590/1982-43272664201601

Smetana, J. G. (2017). Current research on parenting styles, dimensions, and beliefs. Current opinion in Psychology, 15, 19-25. doi: https://doi.org/10.1016/j.copsyc.2017.02.012

Syed, E. U., Hussein, H. S., \& Mahnud, S. (2007). Screening for emotional and behavioural problems amongst 5-11-yearold school children in Karachi, Pakistan. Social Psychiatry and Psychiatric Epidemiology, 42(5), 421-427. doi: https:// doi.org/10.1007/s00127-007-0188-x

Urbano, C., \& Yuni, A. (2016). Psicología y cultura de los adolescentes. Argentina: Editorial Brujas.

World Medical Association (1964). Declaracion de Helsinki. Recuperado de https://www.wma.net/what-we-do/ 